\title{
La Nouvelle Constitution Politique de l'État plurinational (NCPE)
}

Une réponse à l'urgence politique nationale du début du Xxi ${ }^{\mathrm{e}}$ siècle ?

La Nueva Constitución Política del Estado Plurinacional (NCPE). ¿Una respuesta

a la urgencia política nacional de principios del siglo XXI?

\section{Christine Delfour}

\section{(2) OpenEdition}

1 Journals

\section{Édition électronique}

URL : https://journals.openedition.org/agedor/5969

DOI : $10.4000 /$ agedor.5969

ISSN : 2104-3353

Éditeur

Laboratoire LISAA

\section{Référence électronique}

Christine Delfour, "La Nouvelle Constitution Politique de l'État plurinational (NCPE) », L'Âge d'or [En ligne], 13 | 2020, mis en ligne le 01 octobre 2021, consulté le 14 octobre 2021. URL : http:// journals.openedition.org/agedor/5969; DOI : https://doi.org/10.4000/agedor.5969

Ce document a été généré automatiquement le 14 octobre 2021.

L'Âge d'or. Images dans le monde ibérique et ibéricoaméricain 


\section{La Nouvelle Constitution Politique de l'État plurinational (NCPE)}

Une réponse à l'urgence politique nationale du début du Xxi ${ }^{\mathrm{e}}$ siècle ?

La Nueva Constitución Política del Estado Plurinacional (NCPE). ¿Una respuesta

a la urgencia política nacional de principios del siglo XXI?

\section{Christine Delfour}

1 Depuis 300 ans, la Bolivie (se) débat autour d'un modèle national d'intégration des populations indiennes et, en ce début de $\mathrm{xxI}^{\mathrm{e}}$ siècle, autour d'un vivre ensemble multiculturel voire interculturel; 150 ans qu'elle bataille sur le caractère fédéral, centralisé, décentralisé ou d'autonomie de l'État; 80 ans qu'elle négocie la nationalisation ou la privatisation des ressources naturelles. En définitive, 300 ans que la Bolivie vit ou survit - diront certains - dans l'urgence. À chaque crise majeure, son lot de mobilisations sociales quotidiennes, de massacres fortement réprimés, de désordre, de chaos, de violences et d'incertitudes: "País al borde del abismo", "laberinto de tensiones irresueltas", "ausencia de sentido común", "polarización extrema", etc. À chaque crise majeure, sa Constitution. On en dénombre 21 entre 1826 et 2018, même si le nombre ne fait toutefois pas consensus entre les historiens. Certaines d'entre elles ont été modifiées et/ou ratifiées par des Assemblées constituantes, des Congrès ou des conventions; d'autres ne l'ont pas été. L'existence formelle de ces chartes revêt, d'une part, l'apparence d'une résolution de crise et d'un retour au calme et, d'autre part, donne une légitimité au changement politique. Cependant la spirale de la confrontation reprend plus violemment que jamais au tournant $\mathrm{du} \mathrm{xxI}^{\mathrm{e}}$ siècle, accompagnée de son contingent d'affrontements et d'antagonismes.

2 C'est dans le contexte du «Cycle rebelle » (1995-2005) (Guerre de l'eau, Guerre du gaz), que les rapports entre les mouvements sociaux, les partis et l'État se transforment radicalement. Ils sont d'une part, trop puissants pour être instrumentalisés par l'État et, d'autre part, trop multiformes et contradictoires pour revitaliser des partis, qui ont perdu toute crédibilité, et/ou se muer, eux-mêmes, en « partis » - au sens habituel du 
terme. Ce sont les années où la rue est tout et l'État peu de chose, où « la force de la masse et de la multitude en marche $»^{1}$ occupe l'espace public. De porteurs de revendications séculaires, les mouvements sociaux deviennent contestataires, puis de contestataires ils passent à insurgés, pour finalement prétendre au pouvoir. Le triomphe d'Evo Morales, syndicaliste paysan aymara, aux élections de décembre 2005 avec presque $54 \%$ des votes envoie un message clair sur l'urgence de transformer le pays, sur la crise du modèle de domination mis en place par les élites qui se vivent comme blanches, et la nécessité d'une démocratie inclusive.

3 L'Assemblée constituante (2006-2009) devient le principal instrument pour rendre possible ce changement. Elle est alors, dans l'imaginaire collectif, l'espace dans lequel

Bolivianos y bolivianas mestizos, blancoides e indígenas de oriente y occidente, del campo y de las cuidades, (podrían) generar un nuevo pacto social que permitiera la construcción de una sociedad más democrática, justa e igualitaria. ${ }^{2}$

Elle est espace de propositions et d'espérances pour revivifier la démocratie en renforçant la participation citoyenne dans la sphère publique.

Nous pouvons nous demander si la Nouvelle constitution politique de l'État (NCPE), approuvée par référendum en 2009, répond à l'urgence politique des Boliviens indépendamment de leur classe et/ou de leur ethnie ? Donne-t-elle un sens à la réalité factuelle et symbolique de la Bolivie du $\mathrm{xxI}^{\mathrm{e}}$ siècle? In fine, participe-t-elle à la construction du récit national? Pour répondre à ces questions, nous aborderons le texte constitutionnel en tant que macro-récit d'une société dans laquelle l'individu et le collectif génèrent des relations de reconnaissance et d'appartenance. En effet, nous nous inscrivons dans l'approche "néo-constitutionnaliste" du droit et, tout particulièrement dans la perspective de Dominique Rousseau, pour qui la constitution peut donner du sens à la vie individuelle et collective d'un pays quand elle est comprise en tant que récit qui raconte des histoires :

Comme les mythologies des sociétés modernes, les constitutions modèlent le récit de l'histoire et l'oralité politique d'une communauté déterminée, les principes constitutionnels étant la référence à l'imaginaire social le plus immédiat: ils représentent la dimension littéraire et mythologique du droit. ${ }^{3}$

Nous ferons, dans un premier temps, une lecture synthétique des singularités de l'histoire constitutionnelle de la Bolivie afin d'en dégager des invariants susceptibles d'éclairer notre objet, pour, dans un second temps rendre compte des processus utilisés pour aboutir au projet politique plurinational, voire pour résoudre l'urgence.

\section{Un retour sur le passé pour comprendre l'urgence du présent}

7 Force est de constater après une rapide ojeada de l'histoire constitutionnelle bolivienne, donc de 1826 à nos jours, que celle-ci est à la confluence de plusieurs traditions juridiques venues d'ailleurs, principalement d'Europe : un code civil napoléonien, un code pénal espagnol, un aménagement territorial en départements et cantons à la française au XIX ${ }^{e}$ siècle et, très récemment, des propositions d'autonomie empruntées à l'Espagne démocratique ou à l'Allemagne des Landers. À cela ajoutons-y la tradition juridique nord-américaine où la constitution est la règle du jeu de la concurrence politique et sociale : un pacte a minima dans lequel il est conféré aux citoyens une entière autonomie pour le développement égalitaire de leur vie personnelle et pour la 
prise de décisions collectives. La constitution est alors juridiquement supérieure aux autres lois.

8 Un rappel complémentaire au sujet de la tradition juridique venant de la Révolution française nous semble nécessaire: on y aborde le texte constitutionnel en tant qu'incarnation d'un projet politique à la manière d'une entreprise de transformation sociale et politique qui ne se limite pas à poser des règles de jeu. Elle participe directement en conditionnant les futures décisions collectives de la vie économique, sociale, éducative, du travail, etc. Elle régule l'organisation du pouvoir et, en même temps, elle génère des droits et des obligations exigibles. Nous savons tous qu'il n'est pas permis d'ignorer la loi. Bref, la culture juridique de la Bolivie est à la croisée de ces traditions juridiques multiples, dans une sorte de pot-pourri pour reprendre une métaphore utilisée par Ricardo Calla, sociologue bolivien, dans un pays à majorité indienne et à l'histoire coloniale rémanente et singulière.

9 Certes, au niveau continental, paradoxalement, au $\mathrm{xx}^{\mathrm{e}}$ et au $\mathrm{xxI}^{\mathrm{e}}$ siècles, il a fallu en Amérique latine (la Bolivie ne faisant pas exception), laisser de côté en partie les traditions du droit exposées précédemment et façonnées par les textes de Simon Bolivar qui sont les plus nombreux et les plus aboutis en matière constitutionnelle. À titre d'exemples: la Lettre de Jamaïque (6/09/1815), le Discours d'Angostura (15/02/1819), Lettre adressée aux Constituants de la République bolivienne $(25 / 05 / 1826)^{4}$. Comme le signale si joliment le constitutionnaliste Hubert Gourdon, les Indépendances ont déposé les constitutions dans la corbeille de la mariée de la République, mais tout était à inventer ou réinventer à l'aune des épreuves et des rencontres :

Parti depuis les côtes Caraïbes avec Montesquieu et Rousseau en poche, Simon

Bolivar rencontra au hasard des chemins des Andes et de ses haltes guerrières

l'ombre de Hobbes, de son état de nature... et de son Léviathan. ${ }^{5}$

$10 \mathrm{Au}$ cours du xIX ${ }^{\mathrm{e}}$ siècle, la majorité des textes constitutionnels, en Bolivie, répondait à des processus de légitimation politique de gouvernements de facto (caudillos letrados ou bárbaros $\left.{ }^{6}\right)$. Il en fut de même au $\mathrm{Xx}^{\mathrm{e}}$ siècle, quand les États-Unis, soucieux de préserver leur hégémonie et leurs intérêts économiques sur le continent latino-américain imposaient, en pleine Guerre froide et révolution cubaine, des gouvernements de militaires, entraînés à la lutte contre le communisme à l'École des Amériques à Panamá. Ce fut le cas du général René Barrientos en 1964 ou du général Hugo Banzer en 1971. Une convention ratifiait le coup d'état et/ou la rupture démocratique (en 1966-1967 par exemple). Paradoxalement, c'est un gouvernement militaire, celui de ce même général Barrientos qui est à l'initiative de la convocation d'une Assemblée constituante (1966) et de la promulgation d'une nouvelle Constitution en 1967, s'inscrivant ainsi dans la volonté de donner une légitimité à l'illégitimité du coup d'État, tel qu'il est défini par Herbert Klein : un « régime autoritaire, urbain et anti-ouvrier et conservateur, allié de la paysannerie indienne » (Pacto Militar campesino) ${ }^{7}$.

\section{Le « constitutionnalisme social » : une tentative de sortie de crise}

11 La Convention de 1938 et la Constitution de 1967 ont marqué la vie démocratique bolivienne jusqu'à nos jours. Elles nous éclairent sur la problématique qui nous interroge. La Convention de 1938 est une des réponses majeures à la crise provoquée 
par la Guerre du Chaco entre 1932 et $1935^{8}$ et à la résolution de l'urgence dans laquelle se trouvait le pays, contrôlé par la "rosca" qui sert directement les intérêts des Barons de l'étain depuis 1880. Augusto Céspedes qui invente le terme dans les années 1930, le définit ainsi : "Un groupe d'autochtones boliviens et d'étrangers qui, à partir du territoire bolivien, aide le "Super-État" minier à piller le pays en échange d'emplois et de rançons "'. On retrouve une situation comparable à la même période dans d'autres pays d'Amérique latine, mais ce qui fait la spécificité de la Bolivie, c'est la très faible marge de manœuvre financière, éthique et décisionnelle que lui concèdent les Barons de l'étain. «Le grand exploiteur des mines », précise Augusto Céspedes, "a réduit la plutocratie nationale à son strict minimum, à un cercle limité, à une minorité si naine de représentants qui se relaient, que le Super-État lui a fait perdre également sa qualité d'oligrachie ou de bourgeoisie et qu'il l'a réduite au statut d'une rosca misérable dans une nation prolétaire $»^{10}$.

12 Néanmoins, la Convention de 1938 jette les bases de la Révolution nationaliste de 1952 et, pour la première fois, donne un rôle majeur à l'État. Elle est un début de réponse à l'urgence nationale: des représentants des ouvriers, des jeunes et des différents secteurs économiques urbains entrent au Parlement ainsi que des représentants des populations de l'Oriente ${ }^{11}$, de Santa Cruz de la Sierra et du Beni. L'absence de représentation des Indiens et des femmes est, pour la première fois, évoquée. Valentín Abecia Valdivieso dans son ouvrage, Historia del Parlamento" ${ }^{12}$, remarque que: "La Convención del 38 era una especie de insurgencia popular por su ansia de abolir las formas concretas de explotación del país por la rosca minera-feudal." Cette convention fait écho donc à la réalité d'un tissu social complexe dont la Guerre contre le Paraguay et le désordre qu'elle suscita ont fait couver la conscience d'appartenance à une nation bolivienne qui devient réalité, en 1952, avec le Movimiento nacionalista revolucionario $(\mathrm{MNR})^{13}$.

13 Augusto Céspedes ${ }^{14}$ participe à cette Convention comme député du département de Cochabamba. Il en témoigne également dans son ouvrage El Dictador suicida. 40 años de historia de Bolivia :

La Convención del 38, [...], reforma la Carta del 80 en un modo que significó establecer el primer contacto entre la institución escrita y la realidad nativa, incorporando a sus enunciados metafísicos las nociones del régimen económico, social, obrero e indigenal. Se sustituyó el principio de propiedad quiritaria con el de función social; se reconoció al Estado atribución intervencionista y de control en la economía y - como una facultad soberana frente a los escamoteos de la industria minera - se proyectó el monopolio del Estado en el comercio exterior de minerales.

15

14 C'est le « constitutionnalisme social » : l'organisation d'un régime de protection sociale, familiale et culturelle de la population, y compris des paysans. La Convention donne une fonction sociale à la propriété, place l'État comme régulateur et garant des intérêts collectifs ${ }^{16}$ sans entamer pour autant les intérêts individuels qu'il protège. Enfin, l'État défend la souveraineté des ressources naturelles Cette charte est ainsi l'expression de la défense des intérêts des classes populaires et des aspirations des ex-combattants de la Guerre du Chaco. Il s'agit du premier coup d'arrêt au système oligarchique, mais constatons que la majorité de la population (les Indiens), n'est pas encore représentée. Augusto Céspedes qui fut un des orateurs et un des rédacteurs de la Convention l'avoue : "Aquí no está representada la masa indígena." ${ }^{17}$ Quant à Villaroel Claure, viceprésident de la Convention, il déclare que c'est la première étape vers une réforme 
constitutionnelle qui se devra de prendre en compte la réalité bolivienne : "Encarna un nuevo espíritu, es depositaria de una nueva conciencia. [...] representantes nacionales, no es un común poder parlamentario, sino un mandato para plasmar en hechos los designios del alma colectiva." (excluant les Indiens) ${ }^{18} \mathrm{Il}$ insiste également sur le fait qu'une "mosaique d'hommes» de toutes les tendances politiques, et de la classe ouvrière - petits artisans et commerçants -, (aucune mention des Indiens), jeunes et animés par un esprit de rénovation et de transformation ${ }^{19}$ participe à ce projet qui aurait dû aboutir à la rédaction d'une nouvelle constitution. Les aléas de la politique bolivienne ne le permirent pas.

15 La polarisation politique dans les années 1940-1950 est très forte : grèves de mineurs et massacre de Catavi en 1942, répression brutale, coup d'État militaire de Gualberto Villaroel, mobilisations constantes du mouvement ouvrier dans le jeu politique et constitution d'organisations indiennes ${ }^{20}$ en lutte pour une amélioration des conditions de travail ${ }^{21}$, guerre civile en 1949 , insurrection populaire victorieuse du Mouvement Nationaliste Révolutionnaire le 9 avril $1952^{22}$. Le gouvernement du MNR qui accède au pouvoir prend une série de mesures qui bouleversent la vie de la nation: l'établissement du suffrage universel ${ }^{23}$, l'obligation pour les paysans de s'organiser en syndicats, la réforme agraire sur l'Altiplano et dans la vallée de Cochabamba, la reconnaissance des Indiens comme paysans, producteurs, consommateurs, parties prenantes de la modernisation etc.

16 En revanche, malgré l'affirmation de l'égalité des droits et des devoirs, par exemple, entre les époux et leurs enfants ${ }^{24}$, malgré l'existence d'un statut agraire et paysan, l'État ne reconnaît l'Indien qu'en tant que citoyen oblitérant toujours son altérité, ses us et coutumes, ses langues, son appartenance communautaire, etc. C'est un État "importé », universaliste, modernisateur. Il s'agit d'un modèle d'État inachevé qui, d'une part, "libère » la Nation et d'autre part, la «confisque ». Sur la question de l'ethnicité, l'Indien est «invité» dans le dialogue national. L'appartenance communautaire et l'identité indienne ne jouent pas encore un rôle mobilisateur.

Dans l'histoire des Constitutions boliviennes et ce jusqu'en 1994, l'Indigène " n'existait pas ». Il était "invisible», "abstrait», peu ou pas valorisé historiquement et politiquement. Il n'y a aucune trace de la reconnaissance de ses droits ancestraux. On n'admet ni son histoire, ni sa langue, ni ses formes d'organisation économique et sociale. Il est comme "en dehors de l'histoire» bolivienne, monoculturelle et uninationale. L'Etat, machine bureaucratique détentrice du monopole de la violence (dans le sens de Max Weber,) s'est superposé presque toujours, en Bolivie, à la société et à ses institutions vernaculaires.

\section{Vers un État multiethnique et pluriculturel}

Dans les années 1970-1980, le Mouvement Katariste remet en question le modèle de métissage universel du MNR et réaffirme l'identité indienne: « Nous ne sommes plus les paysans de 1952 », est-il précisé dans le Manifeste de Tiawanaku de $1973^{25}$ : «On nous a réduit à être de simples paysans. On nous a ôté notre identité de peuple aymara. On nous a dit que nous serions libres si nous renoncions à être indiens. Nous avons essayé. Mais ce n'est pas vrai. Soyons à nouveau Aymaras!» Ce n'est que dans les années 1990 que l'indianité est officiellement prise en compte, voire récupérée, par le 
gouvernement de Sánchez de Lozada (1994) alors qu'elle s'inscrit, en même temps, dans un vaste mouvement international de reconnaissance des droits des peuples indigènes.

Les organisations indiennes, les institutions internationales (Nations Unies ${ }^{26}$, Groupe de Contadora, les ONG (Conseil mondial des Églises), les grands blocs régionaux (Union européenne, etc.) exercent une forte pression sur les gouvernements latino-américains (dont le gouvernement bolivien) pour la reconnaissance de la pluriculturalité et de la multiethnicité des populations. L'Équateur, le Pérou, la Colombie et la Bolivie se plient aux injonctions des organismes internationaux et modifient les textes constitutionnels ${ }^{27}$.

La démocratisation de l'éducation, l'accès des Indiens à des postes de responsabilité, notamment dans l'administration, l'abandon par les mouvements et des partis politiques d'opposition du paradigme « de classe » en faveur d'un paradigme qui prend en compte l'ethnicité poussent aussi l'appareil bureaucratique et la classe dirigeante à modifier la Constitution en 1994. L'État bolivien se déclare «multiethnique et pluriculturel » (Art.1). Les droits des Indigènes et de leurs « autorités naturelles » sont reconnus (Art.171). À ce moment-là, cette reconnaissance, certes timide, fut considérée comme une des plus extraordinaires avancées de l'histoire de la République. C'est sans aucun doute vrai mais, dans une logique multiculturelle ou, dit d'une autre façon, à partir du libéralisme multiculturel. C'est la reconnaissance de la diversité, mais il n'y a aucun changement dans la matrice centrale de la domination et de l'exploitation qui est, en définitive, le cœur constitutif de l'État et de la société. En réalité, malgré quelques réformes comme la Loi de Participation Populaire (LPP) ${ }^{28}(1994)$ et la Loi de décentralisation (1995), les attentes des populations "originaires» ne sont pas satisfaites. La polarisation est croissante.

21 C'est face à cette désagrégation aux facettes multiples que se mobilisent des acteurs nouveaux, sur des revendications concrètes, selon des modalités qui rompent avec les vieilles pratiques syndicales et qui rejoignent des pratiques communautaires traditionnelles. Ce sont des femmes, des cocaleros (paysans indiens producteurs de coca), des membres d'associations de quartiers (juntas vecinales). Les revendications relèvent de l'emploi, de la qualité de la vie quotidienne (par exemple, le droit de l'eau) mais aussi de l'intérêt national (maitrise des ressources énergétiques, gas, pétrole, lithium). Les nouvelles modalités d'organisation se nourrissent des pratiques traditionnelles de représentations et de prises de décisions (ayllus). Il s'agit du «Cycle rebelle ».

Álvaro García Linera, sociologue et vice-président de l'État plurinational de Bolivie (2005-2019), ne parle pas de mouvement identitaire strictement « indianiste », même si cette composante est essentielle, mais d'un ensemble complexe caractérisé par l'« irradiation de l'identité ethnique à d'autres sphères et espaces subalternes qui articulent luttes de classes et luttes ethniques ${ }^{29}$. La dimension ethnique se réarticule aussi dans des ensembles systémiques : régional, genre et classe entre autres. García Linera précise :

Ces ensembles n'ont pas à être homogènes, ni harmonieux, ni équilibrés étant donné que toutes sortes d'antagonismes qui viennent des contextes dans lesquels ces ensembles sont intégrés ont une influence. Bien que l'ethnicité ait des dynamiques propres, la discrimination est le résultat et, en même temps, le levier reproducteur de mécanismes d'exploitation et de domination qui nous obligent à penser l'ethnicité sans la réduire à sa variable strictement culturelle. ${ }^{30}$ 
Il existe une double, triple, voire une quadruple identité et appartenance organisationnelle sans qu'aucune ne prévale sur les autres dans le mouvement social, de telle façon que le Cycle rebelle se nourrit de la lutte des classes, du nationalisme indigène et révolutionnaire, des revendications ethniques et de l'indianisme, sans oublier le puissant caractère anti-impérialiste du mouvement des cocaleros et des luttes de quartiers, adossé au combat contre la privatisation des services de base et l'émergence d'une nouvelle génération de droits humains comme le droit de l'eau. On est aux antipodes de la stance nationale révolutionnaire du MNR, fondée sur des explications unidimensionnelles.

\section{L'Assemblée constituante de 2006 : la bataille des imaginaires}

$\mathrm{Au}$ seuil $\mathrm{du} \mathrm{xxI}^{\mathrm{e}}$ siècle, la bataille ouverte des imaginaires fait rage: l'imaginaire unidimensionnel, libéral «colonial» dominant depuis la fondation de la République, imposé par une élite pour qui l'état est l'instrument d'un pouvoir associé à des lois injustes "que no se respetan, están mal diseñadas y benefician a una minoría" ${ }^{31}$; et un imaginaire de l'auto-représentation et du contrôle social. Certes, les Boliviens sont convaincus que le combat pour le changement doit se faire dans le cadre constitutionnel et jamais en dehors de la Constitution:

A pesar de los conflictos estamos optimistas, queremos cambio y Bolivia está cambiando. La democracia somos todos: esto implica conflicto y concertación, participación y control social. Los recursos naturales pertenecen a todos y su industralización debe ser la base de nuestro desarrollo. Somos aymaras, quechuas, cambas y collas: somos diversos pero ante todo somos bolivianos. La Asamblea constituyente es participación ciudadana y justicia social, es el escenario de un nuevo pacto social. Queremos nuevas leyes justas y adecuadas y sobretodo que la nueva Constitución se cumpla para que incida en nuestras vidas. ${ }^{32}$

La Constitution et le processus de réformes se trouvent alors au centre de l'imaginaire du changement en démocratie que les Boliviens appellent de leurs voeux en élisant, à la majorité absolue (55\%), le 4 décembre 2005, Evo Morales, président de la République, un Indien, aymara, syndicaliste cocalero. Le projet d'une Assemblée constituante entre alors dans le langage quotidien. La Constitution est l'instrument qui doit en finir avec l'état d'urgence structurelle du pays, "a través de normas de convivencia pactadas fortaleciendo una noción de bolivianidad que remita a la idea de unidad en la diversidad"33. C'est l'opportunité pour trouver une sortie au «labyrinthe des tensions irrésolues ». C'est la cristallisation d'un nouveau ideario autour de sujets aussi fondamentaux que les lois, la souveraineté nationale, la citoyenneté, la nation, les autonomies. Bref, c'est un nouveau «sens commun ». Mais c'est aussi un "processus participatif qui doit bénéficier aux Boliviens les plus nécessiteux $»^{34}$ dans un contexte de concertation et d'accords assez larges pour garantir un minimum de principes et de stabilité politiques.

Pour la première fois dans l'histoire bolivienne, des représentants des populations indiennes, démographiquement majoritaires ${ }^{35}$, sont des constituants. On peut parler d'une Assemblée constituante inclusive. Dans une enquête réalisée en $2008,56 \%$ des constituants s'auto-définissent comme membres d'un peuple "originaire», 32\% quechuas, $17 \%$ aymaras, $7 \%$ appartenant aux autres peuples originaires. De façon plus 
générique et certes au prisme de la race, la grande majorité se considère à 70,8\% métis, $26,7 \%$ indigènes et à peine $3 \%$ blancs. Seuls 3 constituants se disent «boliviens ». Des $2 / 3$ de ceux qui se disent aymaras, $65 \%$ se définissent également "indigènes » alors que, chez les quechuas et chez les autres peuples originaires minoritaires, 59\% et $69 \%$ respectivement se reconnaissent également métis. Ceci n'a rien de surprenant et c'est même cohérent avec l'imposition par la Révolution de 52 d'une catégorie homogène et politiquement correcte face à l'émergence des peuples originaires: le métis ou cholo. Des constituants qui s'auto-identifient blancs, tel Guillermo Richter du MNR, reconnaissent la diversité de l'assemblée et la saluent :

Un logro fundamental fue la participación democrática e inusual a lo largo del proceso. Allí estuvo toda la diversidad cultural del Estado boliviano. Fue muy gratificante encontrarse un nivel de representatividad tan variado. Es un hecho inédito, tomando como referencia lo sucedido en la anterior Convención de 1967, y sin negar la importancia de las transformaciones que se incorporaron y constitucionalizaron en 1993-94. El escenario de aquellos cambios fue más institucional. En cambio, lo de ahora tiene un sentido social y cultural muy rico. Será muy difícil volver a transformer a Bolivia por vías de cambios a la Constitución sin tomar en cuenta estos niveles de representación tan variados, democráticos, plurales y diversos como lo que se vivió en la Asamblea Constituyente. Ese es un logro central. ${ }^{36}$

Nous n'évoquons pas ici les vicissitudes de l'Assemblée constituante, surtout les 4 derniers mois ${ }^{37}$, mais $61 \%$ des électeurs ont approuvé le texte final par référendum (janvier 2009). Le caractère "indígena originario" est l'axe transversal de la Constitution. Il est présent dès le Préambule :

En tiempos inmemoriales se erigieron montañas, se desplazaron ríos, se formaron lagos. Nuestra Amazonia, nuestro chaco, nuestro altiplano, nuestros llanos y valles se cubrieron de verdores y flores. Poblamos esa sagrada Madre Tierra con rostros diferentes y comprendimos desde entonces la pluralidad vigente de todas las cosas y nuestra diversidad como seres y culturas. Así conformamos nuestros pueblos, y jamás comprendimos el racismo hasta que lo sufrimos desde los tiempos de la colonia. El pueblo boliviano, de composición plural, desde la profundidad de nuestra historia [...] construimos un nuevo Estado. Un Estado basado en el respeto e igualdad entre todos, con principios de soberanía, dignidad, complementaridad, solidaridad, armonía en la distribución y redistribución del producto social, [...] el Estado Unitario Social de derecho Plurinacional Comunitario [...]. Nosotros, mujeres y hombres a través de la Asamblea Constituyente y con el poder originario del pueblo, manifestamos nuestro compromiso con la unidad e integridad del pais. ${ }^{38}$

Le Préambule explicite un nouveau récit national. Il insiste sur la cosmovision et la spiritualité des peuples originaires, plein de résonances cosmiques et de la Nature en passant par la pluralité des choses et la diversité des êtres et des cultures. Il déclare « la fin de l'État colonial, républicain et néoliberal» pour construire un État d'un type nouveau où domine la recherche du "vivir bien", la suma qamaña andine, qui se perçoit également chez les peuples des "tierras bajas" de l'Amazonie. Le droit est alors plus qu'un ensemble de normes, c'est également le discours sur les normes (c'est-à-dire le "discours de ceux qui utilisent le droit») pour reprendre les termes de Dominique Rousseau ${ }^{39} \mathrm{et}$ « sur les idées, croyances et valeurs qui circulent dans la société civile qui quotidiennement recrée et donne vie aux catégories, croyances, figures et fictions du monde juridique $»^{40}$. La Nouvelle Constitution Politique de l'État (NCPE) fait partie du macro-récit bolivien. Elle donne un sens à la fois à la réalité factuelle et symbolique de la société dans laquelle l'individuel et le collectif génèrent des relations de reconnaissance et d'appartenance. Enfin, la Constitution sert à ancrer, à conserver et à 
renouveler une identité communautaire et elle est, en même temps, un moyen de transformation sociale et culturelle. Est-elle une réponse à l'urgence sociétale ?

L'article 2 de la Constitution justifie et légitime le rôle de l'indigène originaire dans la Bolivie nouvelle en mettant l'accent sur la dette historique et culturelle des classes dominantes envers eux, érigeant ces peuples aux racines précoloniales au rang de naciones au même titre que la nación boliviana, expression locale de l'Etat-nation :

Dada la existencia precolonial de las naciones ${ }^{41} \mathrm{y}$ de los pueblos indigena originario campesinos y su dominio ancestral sobre sus territories, se garantiza su libre determinacion en el marco de la unidad del Estado, que consiste en su derecho a la autonomia, el autogobierno, a su cultura, al reconocimiento de sus instituciones y a la consolidación de sus entidades territoriales, conforme a esta Constitución y la ley ${ }^{42}$.

Quelle signification donner aux trois qualificatifs des peuples «indígenas originario campesinos » retenue par les Constituants ? À quels groupes humains correspondentils? À quels territoires appartiennent-ils? Il est évident que l'expression "pueblo indígena" fait allusion à l'identité, à la culture, à l'organisation sociale et aux coutumes communes alors que "originario" exprime le principe de la pré-existence des peuples, des descendants des groupes sociaux et des ethnies qui peuplaient le territoire actuel de la Bolivie bien avant la colonie et la fondation de la République et qui surent maintenir vivantes leur identité, leur culture, leur organisation sociale, leurs systèmes économiques locaux, etc. "Campesino" (paysan) fait référence à une catégorie sociologique et économique, ancrée dans le monde rural.

31 La formule "pueblo originario indigena campesino" n'est toutefois pas excluante. L'article 3 répond au risque de laisser de côté dans la constitution les populations urbaines indiennes et non indiennes face aux populations rurales indiennes et non indiennes, afin de ne pas reproduire, à l'inverse, une société fragmentée, discriminante :

El pueblo boliviano está conformado por la totalidad de las bolivianas y de los bolivianos pertenecientes a las áreas urbanas de diferentes clases sociales, a las naciones y pueblos indigena originario campesinos $\mathrm{y}$, a las comunidades interculturales y afrobolivianas. ${ }^{43}$

Il ne s'agit pas uniquement de l'inclusion des bases historiquement exclues dans le processus symbolique de la refondation du pays mais de trouver un modus vivendi (une charte) pour sortir du labyrinthe historique des tensions non résolues, de l'urgence séculaire. Mais gardons à l'esprit que : "En Bolivia", souligne justement Pablo Mamani Ramírez, "lo indígena constituye casi la sociedad. Es lo urbano y lo rural. Es lo real y lo imaginado. Es población y territorio. Es el devenir y es el pasado. Obviamente esto en articulación con el mundo popular de obreros y sectores medios de la sociedad" ${ }^{44}$. Ceci doit prendre forme dans le texte constitutionnel :

Debe ser parte fundamental del Nuevo Estado en lo politico, en lo social, cultural y económico; o mejor, debe ser lo material de las cosas y el sustrato de la subjetividad colectiva de la forma de hacer y sentir el mundo. Así sería un nuevo Estado, o más propiamente un estado plurinacional. La reducción de la Constitución sería la expression filosófica de la multiplicidad de realidades sociales, historias, de los movimientos sociales, y de actores sociales, básicamente el reconocimiento fáctico de la realidad compleja y a la vez articulada entre sí. De ello es parte de la Juridicción indígena como la administración de justicia según principios y valores de los pueblos indígenas originarios campesinos..$^{45}$ 
Pablo Mamani Rodríguez souhaiterait même aller au-delà de ce texte qui ne le satisfait pas complètement. Le sociologue qualifie cette charte de "constitución intermedia", c'est-à-dire à mi-chemin entre des valeurs libérales (par exemple en économie) et néocoloniale (par exemple, dans le maintien du découpage territorial en département, province et municipalité à côté de l'organisation en municipalités originaire paysannes, ayllus, markas etc.).

La NCPE est une Constitution pluraliste face au monoculturalisme des constitutions précédentes. Elle reconnaît dans l'article 1 que "Bolivia se constituye en un Estado Unitario Social de derecho Plurinacional Comunitario, libre, independiente, soberano, democrático, intercultural, descentralizado y con autonomía." ${ }^{46}$

C'est un État plurinational, reflet de la réalité multiethnique, pluriculturelle, multilingue de la Bolivie qui garantit la possibilité de la libre-détermination des peuples indigènes dans le cadre constitutionnel, la participation de représentants plurinationaux dans l'administration de l'État, l'égalité hiérarchique entre les institutions ordinaires et les institutions indigènes (art. 2). Álvaro García Linera est à l'initiative du concept d'État plurinational «communautaire » faisant écho non seulement à l'organisation territoriale, politique et sociale ancestrale andine mais aussi au système économique, une sorte de « socialisme communautaire ${ }^{47}$.

Los pilares de nuestro Estado y nuestra economía son las comunidades indígenas campesinas y el movimiento obrero organizado [...] un brazo emerge del capitalismo desarrollado, el brazo obrero, y el otro, el brazo indígena emerge de lo que no fue destruido por el capitalismo. La ciencia y la tecnología procedentes del capitalismo y, el mundo agarrio comunitario, que resistió al capitalismo por 500 años, están aquí, pueden y deben irradiarse, expandirse y universalizarse. El socialismo comunitario será la comunidad agraria a nivel planetario, tenemos la semilla comunitaria que crecerá y dará frutos poderosos para Bolivia y para el mundo. Pero la vía democrática se garantiza cuando estos dos sectores logran unir y atraer al resto de los sectores sociales en base al ejemplo, la experiencia y los resultados. ${ }^{48}$

La victoire d'Evo Morales et du Movimiento hacia el socialismo (MAS) a trois significations majeures : la recomposition du système des partis, la renovation générationelle et ethnique dans le leadership politique, et la disparition des codes d'exclusion sociale et ethnique. Elle ne suppose pas mécaniquement un changement radical dans la culture politique au sein de l'exécutif. On retrouve les invariants de la gestion nationalpopulaire : le leadership charismatique héritage des caudillos, l' hyperprésidentialisme qui se manifeste dans la maîtrise par le président en exercice du processus constituant ${ }^{49}$ et de sa volonté de perdurer au pouvoir, tout en proclamant l'essence démocratique du texte constitutionnel ${ }^{50}$, la faiblesse des partis politiques traditionnels, le fonctionnement en réseaux hiérarchisés, la mobilisation des secteurs populaires parfois les uns contre les autres ${ }^{51}$, etc. On remarque néanmoins une avancée capitale: la transformation du profil social des fonctionnaires ${ }^{52}$. Ils sont en majorité indiens et la présence des femmes est manifeste. Les nouveaux serviteurs de l'État sont plus jeunes, ont un meilleur niveau de formation et de revenus que leurs parents et ils appartiennent majoritairement à une organisation sociale ${ }^{53}$. Les responsabilités qu'ils assument dans la construction d'un État plurinational se voient renforcées par leur identité indienne. L'expression $\mathrm{du}$ changement est également linguistique: la disparition du terme "République » dans le texte constitutionel est le symbole de la refondation du pays et $\mathrm{du}$ nouveau récit national. Les «classes populaires » ont 
remplacé les "classes moyennes" sans distinction de race. Le processus de « décolonisation de l'État » est en marche.

La NCPE répond aux demandes des Autonomies régionales, légitime une nouvelle génération de droits tant collectifs qu'individuels, proclame la souveraineté nationale sur les ressources naturelles, etc. Bref, la Nouvelle Constitution Politique de l'État change les paramètres de la gouvernabilité, déplace la représentation des partis traditionnels vers des groupements citoyens ou des sujets collectifs (communautés indiennes). Elle crée des espaces informels qui supportent la pression des acteurs sociaux dotés d'une capacité de mobilisation multiple (contrôle social). Elle structure la réalité sociale de la Bolivie du début du $\mathrm{XxI}^{\mathrm{e}}$ siècle, participe en tant que narration au macro-récit (pluri) national et répond, comme en un instantanné, à l'urgence sociétale du moment. En tant que narration, le texte constitutionnel est doté d'une "plasticité » surprenante avec des termes à forte indétermination et grande imprecision démocratie interculturelle, démocratie communautaire, démocratie directe et participative, diversité ethnico-culturelle, pluralisme économique - avec des clauses capables d'être adaptées dans leur signification et leur portée à de nouveaux besoins ou de nouvelles urgences.

\section{NOTES}

1. Fernando Mayorga, Antinomias. El azaroso camino de la reforma política, Cochabamba, CESU-UMSS, 2009,289 p.

2. Guido Riveros Franck, "Los consensos de la Constituyente", Temas de reflexión y Debate, $\mathrm{n}^{\circ}$ 17, p. 3, Fundación Boliviana para la Democracia Multipartidista (fBDM), La Paz, 2006.

3. Dominique Rousseau, Le Droit dérobé, Paris, Éditions Montchrétien, 2007, p. 15.

4. Simón Bolivar, Obra política y constitucional, Madrid, Editorial Tecnos, 2007

5. Hubert Gourdon, «Les trois constitutionnalismes de Simon Bolivar », Cahiers de l'Amérique latine, n²9-39, IHEAL, Paris, p. 249-261.

6. Expressions de l'historien Alcides Arguedas, Obras Completas, t. I "La fundación de la República", Madrid, Aguilar, 1959.

7. Herbert Klein, Orígenes de la Revolucion nacional boliviana. La crisis de la generacion del Chaco, La Paz, Libreria Juventud, 1968, p. 299.

8. Guerre du Chaco entre la Bolivie et le Paraguay (1932-1935) suite à l'occupation par les Paraguayens des départements du Sud de la Bolivie (Chaco Boreal). En trois années de guerre, 65000 Boliviens périssent, soit environ 15\% de la population, majoritairement des Indiens, enrôlés de force. Le traité de 1938, trois années après la fin du conflit, ratifie la perte d'un cinquième du territoire bolivien au profit du Paraguay, mais in fine des compagnies pétrolières transnationales hollandaise et nord-américaine.

9. Augusto Céspedes, El dictador Suicida, 40 años de historia de Bolivia, Santiago de Chile, Editorial Universitaria, 1956, p. 13.

10. Ibid., p. 14.

11. Oriente ou Tierras Bajas, siruée à l'Est de la Bolivie, région tropicale du bassin amazonien. 
12. Valentín Abecia Valdivieso, Historia del Parlamento, t. III, La Paz, Presidencia del Congreso Nacional Edit., 1999, p. 32.

13. La révolution de 52 (1952-1964) douze années de relations singulières entre civils, militaires, paysans et ouvriers (Co-gouvernement Centrale ouvrière bolivienne - COB - et MNR, Pacte Miltaire-paysan).

14. Augusto Céspedes, romancier et homme politique a brossé magistralement dans les romans Sangre de mestizos et El Pozo la fraternité et la solidarité entre Indiens et non Indiens pendant le conflit auquel il participa comme officier.

15. Augusto Céspedes, El Dictador suicida..., op. cit., p. 168-171.

16. Un ministère du Travail est créé; à sa tête, Walter Alvarez, ouvrier, imprimeur, de la Fédération ouvrière du Travail (FOT). Un code du Travail et un décret de sindicalisation obligatoire sont votés par le Parlement. Parmi les nombreux ouvrages à consulter sur cette période, voir tout particulièrement Klein Herbert, Orígenes de la Revolucion nacional boliviana, Juventud editores, La Paz,1968.

17. Augusto Céspedes, Redactor de la Convención de 1938, ANB, Sucre, t. I, p. 175.

18. Villaroel Claure, Redactor de la Convención de 1938, ABN, t. I, p. 85.

19. Ibid., p. 215.

20. La Confederacón Sindical de Trabajadores de Bolivia (CSTB) donne son soutien au mouvement indien en langue quechua qui se réunit, à Sucre, à deux occasions en 1942 et 1943. Premier Congrès Indigène National, à La Paz, en 1945.

21. Il est à noter que les Indiens ne demandent pas encore une redistribution des terres, une réforme agraire mais seulement l'abolition du servage. Décret du 15 mai 1945 (abolición del Pongueaje y mitanaje). Voir James Malloy, The Incompleted Revolution, Pittsburg University Press, 1970, p.164.

22. Les femmes obtiennent le droit de vote aux élections municipales (Convention de 1945).

23. Respectivement en avril et août 1952

24. Constitución Política del Estado de 1967: “Todos los hijos sin distinción de origen tienen iguales derechos y deberes respecto de sus progenitores”, La Paz, ANB, art. 195.

25. Manifiesto de Tiahuanaku, La Paz, Los Amigos del Libro, 1973, 24 p. L'échec partiel du projet modernisateur de la réforme agraire a entraîné, dans les années 1979-1980, une radicalisation du syndicalisme paysan : le mouvement katariste-indianiste, aymara sous le leadership de Raimondo Tambo et Genaro Flores. L'indianisme katariste est un courant politico-cultuel de reconstructionréinvention de l'identité nationale-indigène par une élite intellectuelle-syndicale de paysans migrants aymaras à la ville de La Paz. Pour une analyse exhaustive du mouvement katariste, voir Javier Hurtado, El Katarismo, La Paz, HISBOL, 1986, 340 p. ; Alvaro García Linera (Coord.), Marxa Chavez León, Patricia, Costas Monje, Sociología de los movimientos sociales en Bolivia. Estructuras de movilización, repertorios culturales y acción política, La Paz, Plural Editores, 2004, 688 p. ; Silvia Rivera Cunsiquanqui, Oprimidos pero no vencidos. Luchas del campesinado aymara y qhechwa 1900-1980, La Paz, HISBOL-CSUTCB, 1984, 201 p.; Esteban Ticona Alejo, Organización y liderazgo aymara, 1976-1996, La Paz, Plural Editores, 2000, 214 p.

26. Les Nations Unies déclarent l'année 1992, année des Droits des populations originaires.

27. Christian Gros (dir.) Être indien dans les Amériques, Spoliations et résistance, Mobilisations ethniques et politiques du multiculturalisme, Paris, IHEAL, 2006, $351 \mathrm{p}$.

28. La Loi de Participation Populaire (LPP) renforcée par la Loi des Municipalités (1999) dote les municipalités de pouvoir politique et des ressources financières les transformant en acteurs à part entière du développement local. Elle renforce également leur légitimité démocratique en permettant l'inclusion (timide il est vrai) de leaders originaires et de représentants de communautés indiennes au sein des conseils municipaux. La Loi de décentralisation administrative (LDA) ajuste le rôle des exécutifs des départements (équivalent de nos régions) au 
nouveau découpage administratif des municipalités. Ils deviennent ainsi des sortes de médiateurs entre l'échelon national et l'échelon local.

29. Álvaro García Linera, "Multitud y comunidad, la insurgencia social en Bolivia", Revista Chiapas, Mexico, $\mathrm{n}^{\circ} 11$, Era, 2002. Le concept d'irradiation a été théorisé par René Zavaleta Mercado, Las masas de noviembre, La Paz, Juventud, 1983.

30. Cité par Pablo Iglesias Turión et Jesús Espadasin Lopez, Bolivia en Movimiento. Acción colectiva y poder político, Madrid, El Viejo Topo, 2007, p. 57.

31. Programa de Naciones Unidas para el Desarrollo (PNUD). Bolivia: Encuesta para el desarrollo humano, El estado de la opinión, los bolivianos, la Constitución y la Constituyente, La Paz, 2007. 3500 personnes interrogées au début de l'enquête, 33 entretiens individuels, puis 2100 personnes différentes interrogées à trois reprises dans les zones urbaines et rurales entre juin 2006 et mai 2007.

32. Ibid., p. 24.

33. Ibid., p. 70.

34. Enquête PNUD 2007.

35. Dans le recensement de 2001, 30,07\% des plus de 15 ans s'identifient quechuas, $25,2 \%$ aymaras, 1,6\% guaranis, 2,2 \% chiquitanos, $0,9 \%$ mojeños et $1,4 \%$ à un des 22 peuples indigènes minoritaires soit $62 \%$ de la population. Xavier Albó, Carmen Beatriz Ruiz (coord.), “Autonomías Indígenas en la realidad boliviana y su nueva Constitución", T'inkazos, Revista Boliviana de Ciencias Sociales, La Paz, 2008, n²3/24.

36. Guillermo Richter, entrevista colectiva T'inkazos, La Paz, op.cit, p.16.

37. La nouvelle constitution bolivienne a fait l'objet de très nombreuses publications. À titre d'exemple, Luis Antezana, Bolivia: historia de las Constituciones, La Paz, Cima, 2006 ; Carlos Böhrt, Carlos Alarcón, Carlos Romero, Hacia una Constitución democrática, viable y plural. Tres miradas, La Paz, fMDM, Friedrich Ebert Stiftung, abril de 2008, 292 p. ; Carlos Böhrt Irahola, Silvia Chávez Reyes, Andrès Torrez Villa Gómez, Puentes para un diálogo democrático, La Paz, fMDM, Friedrich Ebert Stiftung, febrero de 2008 ; Fundación Boliviana para la Democracia Multipartidista (fBFM), Los Consensos de la Constituyente, La Paz, Serie : temas, reflexión y debate, $\mathrm{n}^{\circ} 17$, noviembre de 2007 , 59 p. ; Roberto Laserna, Henry Oporto, Luis Verdesoto, Marité Zegueda, Constitución y poder político, apuestas de reforma en Bolivia, La Paz, CERES, USAID, Plural editores, 2007 ; Pablo Mamani Ramírez, "Entre lo liberal y lo comunitario. Un Estado realmente plurinacional?", Le Monde Diplomatique, La Paz, año 1, $\mathrm{n}^{\circ} 9$, diciembre de 2008; Carlos Romero, El proceso constituyente boliviano. El hito de la Cuarta marcha de tierras bajas, Santa Cruz, CEJIS, 2005 ; Ximena Soruco Sologuren, Apuntes para un estado plurinacional, La Paz, Vice-presidencia del Estado plurinacional, Editoral Mava, 2011.

38. Nueva Constitución Política del Estado (NCPE), La Paz, U.P.S, 2009, p. 3.

39. Dominique Rousseau, Le Droit dérobé, ...op. cit, p. 22.

40. Ibid.

41. C'est la référence directe à la Déclaration sur les Droits Indigènes des Nations Unies (2007) dont l'article 9 reconnaît que « les peuples et personnes indigènes ont le droit d'appartenir à une communauté ou une nation indigène ».

42. Nueva Constitución... op. cit, p. 5.

43. Ibid., p.5

44. Pablo Mamani Ramirez, El estado neocolonial: una mirada al proceso de la lucha por el poder y sus contradicciones en Bolivia, La Paz, Rincón Ediciones, 2017, 252 p.

45. Ibid., p.107.

46. Nueva Constitución Política del Estado, op. cit., p. 5.

47. Vice presidencia del Estado plurinacional, Presidencia de la Asamblea legislativa plurinacional. Entrevista al Vicepresidente del Estado plurinacional, Cuidadano Álvaro García Linera, Programa "El Pueblo es Noticia", Canal 7-Red Patria Nueva, 7 de febrero de 2010, 
"Socialismo comunitario. Un aporte de Bolivia al mundo", Reflexiones sobre la coyuntura, La Paz, Año 3, n5, D.L.XXXXX p.O.

48. Ibid.

49. Evo Morales comme Hugo Chávez, au Vénézuela, $(2007,2009)$ renoue avec la tradition constitutionnelle bien connue au temps du caudillisme, celle d'un "presidencialismo constituyente" : modifier, assouplir la clause de rééligibilité immédiatement et indéfiniment reconductible par exemple (référendum du 21 février 2016).

50. En prenant le risque d'une rupture démocratique et du départ précipité de Evo Morales et de l'exécutif en exil au Mexique puis en Argentine en novembre 2019 (élections présidentielles).

51. Les cocaleros du Chaparé (fief d'Evo Morales) contre les cocaleros des Yungas (région historique de la culture de la feuille de coca) pour la règlementation de la production de la feuille de coca, par exemple.

52. Ximena Soruco, Daniela Franco, Mariela Durán, Cosmovisión social del Estado plurinacional. Hacia la descolonización de la burocracia, La Paz, Centro de Investigaciones Sociales (CIS, Viceresidencia del Estado), 2014, 312 p.

53. Il est obligatoire de parler l'espagnol et une langue des populations originaires pour se présenter aux concours de la fonction publique et territoriale.

\section{RÉSUMÉS}

Le triomphe d'Evo Morales, syndicaliste paysan aymara, aux élections présidentielles (décembre 2005) avec presque 54\% des votes envoie un message clair sur l'urgence de transformer le pays, sur la crise du modèle de domination et la nécessité d'une démocratie inclusive. L'Assemblée constituante (2006-2009) devient le principal instrument pour rendre possible ce changement. Elle est alors, dans l'imaginaire collectif, espace de propositions et d'espérances pour revivifier la démocratie. La Nouvelle Constitution Politique de l'État (NCPE-2009) répond-elle à l'urgence politique des Boliviens indépendamment de leur classe et/ou de leur ethnie ? Donne-t-elle un sens à la réalité factuelle et symbolique de la Bolivie du xxI ${ }^{\mathrm{e}}$ siècle ? In fine, participe-t-elle à la construction du récit national ? Le texte constitutionnel est abordé ici en tant que macro-récit d'une société dans laquelle l'individu et le collectif génèrent des relations de reconnaissance et d'appartenance. Mais, est-elle la réponse - définitive - à l'urgence sociétale ?

La victoria de Evo Morales, sindicalista campesino aymara, a las elecciones presidenciales (diciembre de 2005) con casi el 54\% de los votos manda un mensaje claro sobre la urgencia de transformación del país, sobre la crisis del modelo de dominación y la necesidad de una democracia inclusiva. La Asamblea constituyente (2006-09) se convierte en la principal herramienta para realizar el cambio. Está presente entonces en el imaginario colectivo como espacio de propuestas y de esperanzas para revificar la democracia. ¿Responde la Nueva Constitución politica del Estado (NCPE-2009) a la urgencia politica de los bolivianos independientemente de su clase y/o de su etnia? Da sentido a la realidad y simbólica a la Bolivia del siglo XXI? ¿A la construcción del relato nacional? Se analiza el texto constitucional como macro-relato de una sociedad en la cual individuo y colectivo generan relaciones de reconocimiento y de pertenencia. Pero, ¿es la respuesta definitiva a la urgencia societal? 
INDEX

Mots-clés : Bolivie, Constitution, urgence, pluri-nationalité, originaire

Palabras claves : Bolivia, Constitución, urgencia, plurinacionalidad, originario

\section{AUTEUR}

\section{CHRISTINE DELFOUR}

Laboratoire LISAA, Université Gustave Eiffel 